\title{
A normal response to injury- an editorial of the natural healing qualities of inflammation
}

\section{Editorial}

Last week, while out for a run, I felt a sharp pain in my knee. Inspection revealed no obvious biomechanical issue, and the pain was intermittent. Still I took it easy until I returned home. While the area where I had felt the pain was swollen, it was not discolored. Discolored areas generally indicate the breakage of capillaries and small blood vessels under the skin. The black and blue areas are caused by red blood cells and other contents of your circulatory system. While bruising is a sign of trauma, swelling or inflammation is a natural part of the body's response to injury. While there are times when the immune response goes haywire, the inflammatory response is a natural part of the tissue healing process. Inflammation is the term that is used to describe abnormal enlargement of a body part, tissue or organ. The inflammation is generally caused by the buildup of fluid in the tissue, which is comprised of blood and its constituents as well as the lymphatic system. The medical term for fluid that has accumulated in the tissue is edema. In my case, because the inflammation was in my knee joint, the medical term effusion is used to describe the buildup of fluid within the joint. While the injury was not serious, had there been a buildup of blood in the joint capsule, which would be indicative of serious injury, the term hemarthrosis is used to describe this form of inflammation. In such injuries, a sample of the fluid is sometimes extracted using a needle. Often, if the swelling is severe within the first few hours, it is probably associated with a buildup of blood and therefore requires investigation by a physician.

Inflammation is an active aspect of the immune system, and is controlled by various organ systems and tissues in response to injuries or infections. The cells of the immune system travel to the site of injury or infection and create the inflammatory response. The signs of inflammation are warmth (the injured area feels warm to the touch), redness, swelling, and most often pain. Not all inflammation is caused by injury, however, and certain long-term conditions may cause inflammation, including asthma, colitis, Crohn's disease, arthritis, nephritis (kidney disease), and vasculitis (inflammation of the blood vessels). There are many different cells involved in the inflammation process and in the immune system and include lymphocytes (white blood cells of different types), proteins excreted from B cells (nonthymus lymphocytes that produce antibodies), neutrophils (another form of white blood cell), monocytes (the largest of the white blood cells created in the bone marrow and destroy invading microbes by engulfing them), eosinophils (white blood cells involved with numerous inflammatory processes, especially allergic reaction), and basophils (contain anticoagulant which prevents blood from clotting too quickly and histamine, which promotes blood flow). And while reducing pain and blood loss should always be the highest
Volume 7 Issue 4 - 2018

\author{
W Sumner Davis \\ Walden University, USA
}

Correspondence: W Sumner Davis, Community Psychologist and Clinical Epidemiologist, Walden University, Oakland, ME 04963, USA, Tel +0I (207) 740-2028,

Email dr.sumner.davis@gmail.com

Received: July 27, 2018 | Published: July 31, 2018

consideration, a recent article published by the Cleveland Clinic suggests that swelling from inflammation plays a key role in healing of soft tissue. The obvious dilemma is, do we attempt to reduce swelling with ice and anti-inflammatory drugs to reduce short term discomfort, and slow down the healing process? Moreover, how does the use of anti-inflammatory medications impact the ability of the body to heal naturally?

A study published in the journal of the Federation of American Societies for Experimental Biology (FASEB) examined two groups $o$ and unable to produce inflammation. Understandably, the noninflammatory mice were unable to heal injuries to muscle tissue, suggesting that the inflammation process is far more complicated, and perhaps far more necessary than previously known. The Cleveland study also found that macrophages that help clear away damaged cells due to injury, initiating the repair process that triggers inflammation, also produce insulin-like growth factor-one, enhancing muscle tissue reconstruction. In the end, only your doctor can best advise you on how to treat your injury, either through medication, physiotherapy, or rest. But from minor inflammations it may be better to avoid the ice and NSAIDS and allow your body to heal naturally. If your injury is chronic, or if the inflammation lasts more than a few days, it is an indication that the injury may not be healing; continual inflammation suggests the injury is still present and a physician or healthcare practitioner should be consulted. Whether or not the injury requires medical treatment is ultimately a decision you have to make.

\section{Acknowledgements}

None.

\section{Conflict of interest}

The author declares that there is no conflict of interest. 